\title{
At the forefront of thought: The effect of media exposure on airplane typicality
}

\author{
LAURA R. NOVICK \\ Vanderbilt University, Nashville, Tennessee
}

\begin{abstract}
The terrorist attacks of September 11,2001 provided a unique opportunity to investigate the causal status of frequency on typicality for one exemplar of a common conceptual category-namely, the typicality of airplane as a member of the category of vehicles. The extensive media coverage following the attacks included numerous references to the hijacked airplanes and to the consequences of suspending air travel to and from the United States for several days. The present study, involving 152 undergraduates, assessed airplane typicality at three time points ranging from $5 \mathrm{~h}$ to 1 month after the attacks and then again at 4.5 months after the attacks. Airplane was judged to be a more typical vehicle for 1 month following the attacks, relative to a baseline calculated from data collected yearly for 5 years preceding the attacks. By 4.5 months, however, typicality was back to baseline.
\end{abstract}

Rosch's (1975) seminal article demonstrated that some category members are considered to be better, or more typical, members of a category than others are. For example, cars and trucks are very good members of the category of vehicles, whereas sleds and wheelchairs are not; airplanes and trains fall somewhere in the middle. Rosch and Mervis (1975) found that a strong correlate of this graded structure is family resemblance: Typical category members (e.g., cars) share more features with other members of the category than do atypical members (e.g., sleds). Using artificial categories, Rosch and Mervis (1975) and Rosch, Simpson, and Miller (1976) demonstrated the causal status of family resemblance for determining typicality.

Barsalou (1985) examined family resemblance, ideals, and frequency of instantiation as predictors of graded structure for both taxonomic (e.g., vehicles) and goalderived (e.g., foods not to eat on a diet) categories. Ideals are features that exemplars should have if they are to best serve a goal associated with their category; they tend to be extreme values that may be true of few or no category members. Frequency of instantiation is a subjective estimate of how often one has experienced an object as a member of a particular category. This is a category-

I thank Larry Barsalou for helpful conversations concerning multiple aspects of this project. I also thank 26 attendees at the 42 nd Annual Meeting of the Psychonomic Society (November 16-17, 2001) who responded to a questionnaire asking them to guess the direction in which airplane typicality changed on September 11,2001, and to explain their guesses. Their responses helped me think about possible mechanisms underlying alternative predictions concerning the effect of the massive media exposure to airplanes following the terrorist attacks. Correspondence concerning this article should be addressed to L. R. Novick, Department of Psychology and Human Development, Peabody 512, 230 Appleton Place, Vanderbilt University, Nashville, TN 37203 (e-mail: laura.novick@vanderbilt.edu). specific measure of frequency, in contrast to familiarity, which is a category-independent measure-namely, a subjective estimate of how often one has experienced an object across all contexts. In Barsalou's (1985) Experiment 1 , family resemblance, frequency of instantiation, and ideals all made unique individual contributions to predicting typicality for members of taxonomic categories, including vehicles. Any effect of global familiarity was redundant with that of frequency of instantiation. The results of Experiment 2, in which Barsalou (1985) employed an artificial category learning paradigm, demonstrated a causal role for ideals in determining graded structure.

Nosofsky (1988) investigated the causal status of frequency by manipulating the frequency of presentation of a good or poor exemplar of one of two color categories. He found in two experiments that frequency (as well as similarity) causally determined graded category structure.

It is an open question whether frequency also has a causal effect on typicality for conceptual categories. For categories such as those studied by Rosch (1975) and Barsalou (1985), real-world knowledge and implicit theories are crucial. These conceptual variables might overwhelm any effects of frequency. Moreover, given the very large baseline frequencies of instantiation of objects in categories for adults, it is difficult to imagine changing those frequencies significantly in a laboratory study. Nevertheless, Barsalou (1987) has argued quite forcefully that the graded structure of conceptual categories is flexible rather than set in stone (also see Barsalou, 1985; Roth \& Shoben, 1983). The items whose typicality values are most likely to change from one time to another are those, like airplane, that are in the middle of the distribution (Barsalou, 1987; also see McCloskey \& Glucksberg, 1978).

The terrorist attacks of September 11,2001—in which three hijacked airplanes were deliberately flown into the 
World Trade Center and the Pentagon, and a fourth airplane crashed outside of Pittsburgh, PA-provide a unique opportunity to investigate the causal status of frequency on typicality for one exemplar of a common conceptual category - namely, the typicality of airplane as a vehicle. Following the attacks, viewers and listeners were told repeatedly by the news media about the nearly 300 passengers and crew members on these airplanes who never made it to their final destinations, the roughly 8,000 airplanes that were parked on runways, and the tens of thousands of passengers who were stranded at airports all over the U.S., Canada, and elsewhere when air travel to and from the United States was suspended (e.g., "A Day of Terror," 2001). In the present study, I investigated, over the course of 4.5 months, the effect of these events and the media coverage of them on judgments of airplane typicality.

Two contradictory predictions seem sensible. On the one hand, one might expect an increase in the typicality of airplane as a vehicle. The content of the media reports and the repetitive nature of the coverage in the days following the attacks should have increased the availability in memory of airplanes as vehicles, which would increase people's judgments of the frequency of instantiation of airplanes as vehicles (Tversky \& Kahneman, 1973), which would increase typicality. This line of argument is consistent with Barsalou's (1985) correlational data. An alternative explanation is that the media coverage led to a global increase in the availability of airplanes in memory. Even though airplanes were not necessarily linked to a means of transportation (presumably they were also linked to weapons, as will be discussed in the next paragraph), the greater familiarity with this object in general might have led to judgments of greater typicality as a vehicle. I will say more about these alternative mechanisms in the Discussion section.

Alternatively, one might expect a decrease in the judged typicality of airplane as a vehicle. The extensive media coverage might have highlighted the weapon-like properties of airplanes (the hijacked airplanes were used as guided missiles) at the expense of their transportation properties. This could have decreased typicality judgments either because (1) people would think first of the category of weapons, rather than vehicles, when asked to consider airplanes, or because (2) the weapon-related features that airplanes acquired reduced their family resemblance to other types of vehicles.

\section{METHOD}

The independent variable was the amount of time since the terrorist attacks of 9/11/01. The first set of data was collected from 20 Vanderbilt University undergraduates enrolled in Psychology of Thinking who attended class approximately $5 \mathrm{~h}$ after the first attack. Additional sets of data were collected 6 days later (9/17/01) from 42 students in another cognitive psychology class, 1 month later (10/11/01) from 54 students in an introductory statistics class, and 4.5 months later $(1 / 22 / 02)$ from 36 students in another introductory statistics class. To the best of my knowledge, no student completed the experimental task more than once. Airplane typicality at these four time points was compared with a 5-year baseline that came from demonstration data collected yearly in the Psychology of Thinking class from 1996 to 2000 (the first time point came from the same set of demonstrations, collected at exactly the same point in the semester in 2001). All data collected in the cognitive psychology classes were obtained prior to discussion of categorization in class.

Subjects were asked to rank-order 10 vehicles in terms of typicality. Written instructions said to place a " 1 " next to the item that they thought was most typical of the category, a "2" next to the item that they thought was next most typical, etc., down to a " 10 " for the item they thought was least typical. Additional verbal instructions clarified that most typical meant "best example of the category." Each item was listed on a separate line below the category label vehicle. The 10 items, in order of presentation, were (Rosch, 1975, average ratings are in parentheses): airplane $(M=2.64)$, wheelchair $(M=$ $3.68)$, bicycle $(M=2.51)$, bus $(M=1.27)$, car $(M=1.13)$, motorcycle $(M=1.65)$, sled $(M=4.61)$, boat $(M=2.75)$, train $(M=2.15)$, and truck $(M=1.17)$. The rank-ordering procedure was used for simplicity. For the 5 years of baseline data, the correlations between the average ranks and Rosch's (1975) average ratings ranged from 0.94 to $0.95(M=0.95)$.

\section{RESULTS AND DISCUSSION}

\section{Airplane Typicality}

The average typicality rank for airplane ranged from 5.69 to 6.08 across the 5 years preceding the events of $9 / 11 / 01$. The 5 -year grand mean $(M=5.91)$ was taken as the population value against which to compare the average ranks for the present samples.

Immediately after the terrorist attacks, airplane was perceived to be a reliably more typical vehicle $(M=4.90)$ than it had been in the past $[t(19)=-2.23, p<.04, S E=$ $0.453]$. Six days later, students' perceptions of airplanes as vehicles were similar to those observed on the day of the attacks, with a mean of 4.95. As before, this is reliably more typical than the 5 -year baseline $[t(41)=$ $-3.49, p<.01, S E=0.275]$. Airplane typicality remained at the new level on the one-month anniversary of the attacks $(M=4.83)[t(53)=-4.28, p<.001, S E=$ $0.252]$. By 4.5 months after the terrorist attacks, however, airplane typicality had returned to the 5-year baseline $(M=5.97)[t(35)=0.21, S E=0.294]$.

These results are consistent with Barsalou's (1985) hypothesis that frequency of instantiation plays a causal role in determining the typicality of members of taxonomic categories. Whereas airplane previously was ranked as the six th most typical among the 10 vehicles in my list (behind car, truck, bus, motorcycle, and train), during the month following the terrorist attacks it was tied for fourth (with motorcycle), as shown in Figure 1.

It is interesting to consider whether the betweensubjects variability in airplane typicality ranks also changed after the attacks. For example, if most subjects judged airplane to be a more typical vehicle (because of its greater availability in memory) but some thought it was less typical (because it was more like a weapon), the variability in subjects' ranks in the month after 9/11/01 


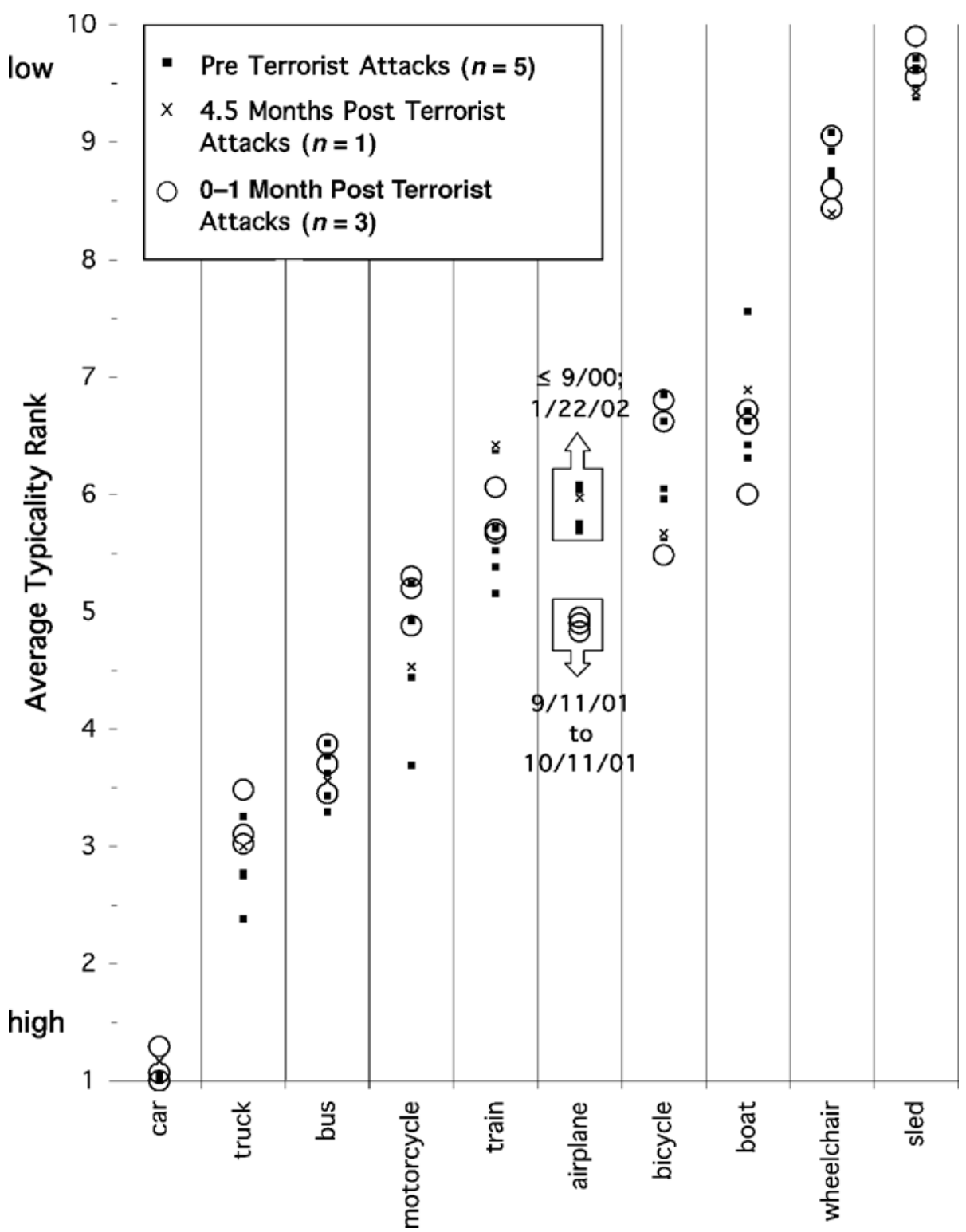

Figure 1. Mean typicality ranks for 10 exemplars of the vehicle category for 5 years preceding the terrorist attacks of $9 / 11 / 01$ and at four time points in the 4.5 months following the attacks.

should have been greater than at the other time points. This does not appear to have been the case. The standard deviations for the 5 baseline years and for 4.5 months after the attacks were 1.98, 2.61, 2.18, 1.96, 2.06, and 1.76 , respectively. For the three time points in the month following the attacks, the standard deviations were 2.02, 1.78 , and 1.85 .

\section{Typicality of Other Vehicles}

The events of 9/11/01 and the subsequent media coverage concerned airplanes but not other types of vehicles. Thus, any effects of these events on the internal structure of this category should be confined to this ex- emplar: The typicality rank for airplane should be the only one to move off the distribution defined by the 5 years of baseline data. To address this issue, I examined the average ranks for each vehicle both before 9/11/01 and on or after that date. These data are shown in Figure 1 . The 10 vehicles are listed in typicality order on the basis of their grand mean ranks from the baseline data. The individual means for each exemplar during this period are indicated by small filled squares. The individual means for each exemplar during the month following the terrorist attacks are indicated by larger open circles. The data for the final time point are indicated by small $\times$ s. It is obvious from this figure that only airplane shows a 
consistent shift in typicality outside the normal range, and only for the month following the attacks.

\section{Frequency of Instantiation Versus Familiarity}

The present results provide the first demonstration of a causal effect of frequency on typicality for a real-world taxonomic category. However, they do not allow a definitive assignment of causal status to greater frequency of instantiation of airplanes as vehicles rather than to greater global familiarity with airplanes (as is suggested by Barsalou's, 1985, correlational data). The extensive media coverage of the terrorist attacks undoubtedly increased both frequencies. Without ratings of both frequency variables collected at baseline and following the terrorist attacks, it is impossible to settle this issue with the present typicality data. Resolution awaits some other (hopefully less negative) real-world event that has a large enough impact on the frequency of instantiation of some object as a member of a category to be noticeable.

\section{REFERENCES}

BARSALOU, L. W. (1985). Ideals, central tendency, and frequency of instantiation as determinants of graded structure in categories. Journal of Experimental Psychology: Learning, Memory, \& Cognition, 11, 629-654.
BARSALOU, L. W. (1987). The instability of graded structure: Implications for the nature of concepts. In U. Neisser (Ed.), Concepts and conceptual development: Ecological and intellectual factors in categorization (pp. 101-140). Cambridge: Cambridge University Press.

"A Day of Terror: Thousands Stranded As the FAA Grounds FlightsAirlines Are Diverted To Canada; Travelers Look for Rental Cars." (2001, September 12). Wall Street Journal, p. A3. Retrieved February 21, 2002, from ProQuest Information and Learning: http://proxy. library.vanderbilt.edu:8080/ogin?url = http://www.umi.com/pqdauto.

MCCloskey,M. E., \& Glucksberg, S. (1978). Natural categories: Well defined or fuzzy sets? Memory \& Cognition, 6, 462-472.

NosOFSKY, R. M. (1988). Similarity, frequency, and category representations. Journal of Experimental Psychology: Learning, Memory, \& Cognition, 14, 54-65.

Rosch, E. (1975). Cognitive representations of semantic categories. Journal of Experimental Psychology: General, 104, 192-233.

Rosch, E., \& MERVIS, C. B. (1975). Family resemblances: Studies in the internal structure of categories. Cognitive Psychology, 7, 573-605.

Rosch, E., Simpson, C., \& Miller, R. S. (1976). Structural bases of typicality effects. Journal of Experimental Psychology: Human Perception \& Performance, 2, 491-502.

Roth, E. M., \& Shoben, E. J. (1983). The effect of context on the structure of categories. Cognitive Psychology, 15, 346-378.

TVErsky, A., \& Kahneman, D. (1973). Availability: A heuristic for judging frequency and probability. Cognitive Psychology, 5, 207232.

(Manuscript received March 13, 2002; revision accepted for publication May 17, 2002.) 